\title{
SGTD: Structure Gradient and Texture Decorrelating Regularization for Image Decomposition
}

\author{
Qiegen Liu ${ }^{1,2}$, Jianbo Liu ${ }^{2}$, Pei Dong ${ }^{3}$ and Dong Liang ${ }^{2 *}$ \\ ${ }^{1}$ Department of Electronic Information Engineering, Nanchang Univerisy, China \\ ${ }^{2}$ Paul C. Lauterbur Research Center for Biomedical Imaging, SIAT, China \\ ${ }^{3}$ School of Information Technologies, the University of Sydney, Australia
}

\begin{abstract}
This paper presents a novel structure gradient and texture decorrelating regularization (SGTD) for image decomposition. The motivation of the idea is under the assumption that the structure gradient and texture components should be properly decorrelated for a successful decomposition. The proposed model consists of the data fidelity term, total variation regularization and the SGTD regularization. An augmented Lagrangian method is proposed to address this optimization issue, by first transforming the unconstrained problem to an equivalent constrained problem and then applying an alternating direction method to iteratively solve the subproblems. Experimental results demonstrate that the proposed method presents better or comparable performance as state-of-the-art methods do.
\end{abstract}

\section{Introduction}

Image decomposition defined as separating an image $f$ as the sum of two independent components $f=x+v$, usually is the first step to the solution of many image processing tasks like inpainting [2], demosaicing [15] and registration [12]. The piecewise smooth function $x$ with quasi-flat intensity plateaus and jump discontinuities is called "cartoon". This component contains main large-scale structure features of the image, and can be used for feature detection, segmentation and object recognition. Another component $v$ usually represented by a small-scale oscillatory function and having some periodicity nature, captures texture and possibly noise, and thus is suitable for solving various texturedepended applications e.g. classification, surface analysis, shape/orientation from texture. Variational approach is the most popular approach to address the image decomposition problem. The common part in this approach is often related to total variation (TV) minimization. The pioneering formulation of such approach was originated from Meyer [9],

${ }^{*}$ Corresponding author: D. Liang dong.liangesiat.ac.cn who suggested starting from the regularization of Rudin et al. (ROF) model, or TV- $L_{2}$ model [14]:

$$
x=\arg \min _{x}\left\{\frac{\mu}{2}\|f-x\|_{2}^{2}+\sum_{i=1}^{n^{2}}\left\|D_{i} x\right\|_{2}\right\}
$$

where $T V(x)=\sum_{i=1}^{n^{2}}\left\|D_{i} x\right\|_{2}$. For each $i, D_{i} x \in R^{2}$ represents the first-order finite-difference of $x$ at pixel $i$ in both horizontal and vertical directions. The term $\left\|D_{i} x\right\|_{2}$ is the variation of $x$ at pixel $i$, and the summation $T V(x)$ is taken over all pixels for a $n \times n$ image. It can be seen that TV- $L_{2}$ model performs decomposition by modeling the cartoon component $x$ with TV semi-norm and using the $L_{2}{ }^{-}$ norm for oscillating features $v=f-x$. Starting from this model, the strategies for improving image decomposition can be divided into three approaches, based on searching the suitable model for texture, cartoon or for both texture and cartoon.

The first approach devotes to modifying the norm on the texture component. Since the ROF model rejects the textures, Meyer defined a new function space $G$, and replaced the $L_{2}$-norm by the $G$-norm. It was proved that $G$ corresponds to a space of oscillating functions, and thus is better suited to model textures. Some approximated norm like $\operatorname{div}\left(L_{p}\right)$-norm [20] and $H^{-1}$-norm (OSV) [11] were developed by following this idea. Particularly, by replacing the $L_{2}$ norm on data fidelity (i.e. texture component) with the $L_{1}$ norm, the importance of TV- $L_{1}$ model in image decomposition has attracted many researchers $[10,3,25]$. Yin $e t$ $a l$. have shown that this model has scale-selection and morphologically invariant properties [25].

The second approach, focusing on modifying the norm on the TV measure, encompasses methods such as weighted least squares (WLS) [5] and $L_{0}$ gradient minimization [22]. Though still depending on gradient images, these two methods differ from the TV- $L_{2}$ decomposition model on regularization term and specific optimization steps, and do not suit texture separation very well. Inspired by the observation that a major edge of cartoon in a local window contributes 
more similar-direction gradients than textures with complex patterns, $\mathrm{Xu}$ et al. [6] introduced relative total variation (RTV) regularization for extracting structure from texture. The experimental results demonstrate that RTV can make main structures stand out by combining general windowed total variation and novel windowed inherent variation.

Unlike the above two approaches that enhance the decomposition by modifying texture norm or cartoon norm individually, a "structural decorrelating" approach for image decomposition has attracted much attention and presented impressive performance recently. The methods in this stream directly predefined the decorrelation measure between the structure and texture components. Shahidi et al. [16] improved the OSV method by introducing the penalty of correlation coefficient between structure and texture components. Szolgay et al. [18] used the angle deviation error (ADE) based orthogonality measure to improve the spatial filter for better separating the cartoon and texture parts. However, the experimental results in [18] showed that the DOSV improvement is limited. Additionally, both DOSV and the ADE-based methods usually have large computational complexity in the optimization stage.

In the present work, a new and yet effective method utilizing the "structural decorrelating" strategy is proposed for image decomposition. Well-decomposed performance is achieved by adding a novel structure gradient and texture decorrelating (SGTD) regularizer to the TV- $L_{2}$ model. Specifically, this proposed regularizer aims to enforce that the correlation between structure gradient and texture components is minimal instead of the correlation between structure and texture components. With regard to the new regularizer, texture and main structure exhibit appropriate decorrelated properties, making them surprisingly decomposable. A robust numerical solver named alternating direction method is proposed to decompose the original highly nonconvex optimization problem into several subproblems, and find the fast and robust solutions.

The rest of this study is organized as follows. In Section 2, we will present our new regularizer and model for image decomposition. In Section 3, an efficient algorithm for solving the proposed model will be derived by applying alternating minimization. Numerical experiments will be provided in Section 4. Finally, concluding remarks and perspectives are sketched in Section 5.

\section{SGTD: Structure Gradient and Texture Decorrelating Regularizer and Model}

In this section, the structure gradient and texture decorrelating (SGTD) regularizer and its corresponding decomposition model will be derived. The intuitive motivation and explanation are presented first. Subsequently the mathematical formulation and model behind this observation are established. Finally, we will reveal the relation and differ- ence between the proposed model and TV- $L_{1}$ model.

\subsection{Structure Gradient and Texture Decorrelating (SGTD) Regularizer}

Intuitively, for a successful decomposition, any given feature in an image should be considered as either a cartoon feature or a textural feature. Therefore, the correlation between the cartoon and texture components of a decomposition should be low, i.e. the range of this random field will consist of values close to zero. This assumption has been adopted in previous work [16, 18]. The straightforward way to model this assumption is to minimize $\sum_{i=1}^{n^{2}}\left|f_{i}-x_{i}\right| \cdot\left|x_{i}\right|$. However, directly calculating the correlation between these two components could cause problem since the texture part has an inherent zero mean while the cartoon does not. In order to help address this issue, we propose an alternative way by minimizing $\sum_{i=1}^{n^{2}}\left|f_{i}-x_{i}\right| \cdot\left\|D_{i} x\right\|$. Different from the straightforward expression, the new model involves finitedifference operation, which makes the gradient images have inherent zero mean since this operation for one pixel could be either positive or negative. Therefore, our proposed regularizer suggests the gradient magnitude of the cartoon and texture component are generated from independent process and thus are uncorrelated.

Theoretically, the SGTD regularizer can be approximately viewed as a window based correlation coefficient with size of two between the texture component and the cartoon component. For example, assume $\left[x_{i-1}, x_{i}\right]$ is a vector representing cartoon variable in a window of size two, and $\left[f_{i-1}-x_{i-1}, f_{i}-x_{i}\right]$ denotes the corresponding texture variables with zero mean, then the absolute value of correction coefficient between the two random variables $X$ and $Y$ is

$$
\begin{aligned}
\left|\rho_{X Y}\right| & =\left|\frac{\operatorname{Cov}(X, Y)}{\sigma_{X} \sigma_{Y}}\right|=\left|\frac{E[(X-\bar{X})(Y-\bar{Y})]}{\sigma_{X} \sigma_{Y}}\right| \\
& =\frac{\left|x_{i}-x_{i-1}\right| \cdot\left|f_{i}-x_{i}\right|}{2 \sigma_{x} \sigma_{f-x}}=\frac{\left|D_{i} x\right| \cdot\left|f_{i}-x_{i}\right|}{2 \sigma_{x} \sigma_{f-x}}
\end{aligned}
$$

Similarly as in DOSV, by assuming that the standard deviations $\sigma_{x}$ and $\sigma_{f-x}$ are not the functions of $x_{i}$, the absolute value of correction coefficient in pixel $i$ can approximate the SGTD regularizer.

Taking the decomposition of image "Barbara" in Fig.1 for example, we can find that the range of the cartoon and texture components may not at the same or near scale (one is 0.8 while the other is 0.5 ). Fortunately, the range of the magnitudes of cartoon gradient and texture components are close to each other (one is 0.4 and the other is 0.5 ), after applying the SGTD regularizer. It can be seen that the magnitude images of cartoon gradient and texture components in Fig.1 (d) and (e) are largely complementary. This observation suggests that these two components are highly uncorrelated and thus are suitable for cartoon + texture decomposition. Moreover, the range of the SGTD values (i.e. 0.035) is 


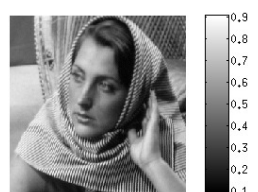

(a)

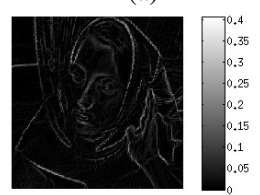

(d)

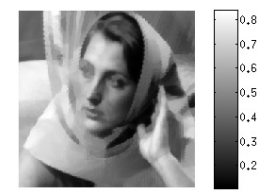

(b)

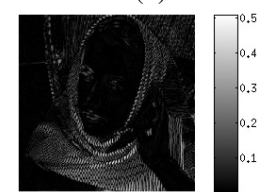

(e)

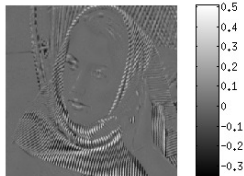

(c)

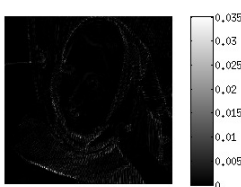

(f)
Figure 1. Numerical validation of the assumption that the small SGTD is necessary for successful decomposition. (a)(b)(c): The input image, cartoon + texture decomposition results. (d)(e)(f): gradient magnitude image of the cartoon component, the magnitude of texture component and their SGTD values.

smaller by an order of magnitude than their original values. This example numerically validates that for an appropriate decomposition of cartoon and texture components, the coherent between the gradient cartoon and texture is small.

\subsection{SGTD Regularization Model}

In this subsection, a new decomposition model involving the SGTD regularizer is presented. Mathematically, the objective function can be expressed as:

$$
\begin{gathered}
x=\arg \min _{x}\left\{\frac{\mu}{2}\|f-x\|_{2}^{2}+\eta \sum_{i=1}^{n^{2}}\left\|D_{i} x\right\|_{2}\right. \\
\left.+\sum_{i=1}^{n^{2}}\left|f_{i}-x_{i}\right| \cdot\left\|D_{i} x\right\|_{2}\right\}
\end{gathered}
$$

where $\mu$ and $\eta$ are the weights. The first term in the cost function enforces data fidelity in the image domain. The second term favors that the structure part to be sparse in the gradient domain. The effect of more powerfully decomposing cartoon and texture is introduced by the third term, which measures the correlated quality between the gradient of structural component and the textural component. Compared to the TV- $L_{2}$ moedel, the new added term alleviates the drawback introduced by only using TV regularizer and makes the proposed model be able to handle images containing complex patterns.

\subsection{The Relation and Difference between SGTD and TV- $L_{1}$ Model}

When $\eta \rightarrow 0$, our model (2) degrades to:

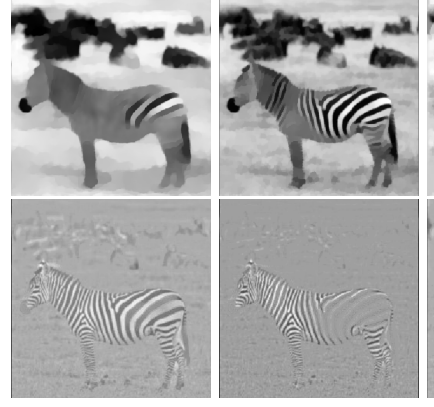

(a) TV- $L_{1}$ (b) TV- $L_{1}$

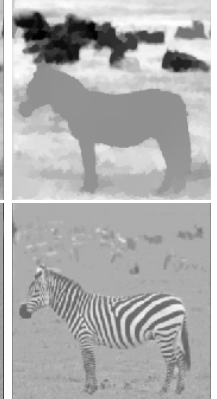

(c) SGTD

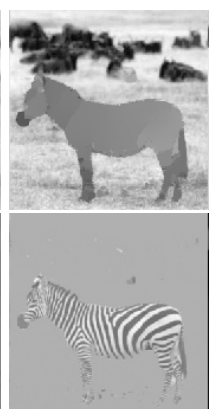

(d) SGTD
Figure 2. The demonstration on the difference between TV$L_{1}$ and SGTD models. The first two columns are obtained by TV- $L_{1}$, where the parameter $\mu$ in (a) and (b) is 0.3 and 0.7 respectively, and the latter two are those by SGTD. The parameter $\mu$ in (a) and (b) is 0.3 and 0.7 respectively, and the parameter pairs $(\mu, \eta)$ in (c) and (d) are $(1500,0.006)$ and $(400,0.003)$ respectively. The perfect separation of various meaningful cartoon and texture patterns may come from the proposed nonlinear reformulation of the model.

$$
\begin{aligned}
x & =\arg \min _{x}\left\{\frac{\mu}{2}\|f-x\|_{2}^{2}+\sum_{i=1}^{n^{2}}\left|f_{i}-x_{i}\right| \cdot\left\|D_{i} x\right\|_{2}\right\} \\
& =\arg \min _{x}\left\{\sum_{i=1}^{n^{2}}\left|f_{i}-x_{i}\right| \cdot\left(\frac{\mu}{2}\left|f_{i}-x_{i}\right|+\left\|D_{i} x\right\|_{2}\right)\right\}
\end{aligned}
$$

In this form, it can be observed that our model can be seen as a weighted TV- $L_{1}$ model with the weight $w_{i}=\left|f_{i}-x_{i}\right|$. Based on this observation, it is natural to conclude that our method may associate to the excellent properties of TV- $L_{1}$ such as contrast preservation and data driven scale selection [25]. Moreover, it allows more freedom and robustness than TV- $L_{1}$, benefited from the nonlinear reformulation. Fig. 2 illustrates one challenging example for decomposition since the texture of the input image Zebra has a wide range of sizes. We can see that the non-textural parts such as slow changes of the gray level values and non-textural parts of the background cannot be appropriately separated from the texture by tuning the parameter of TV- $L_{1}$. This drawback can be overcome by the proposed SGTD method. An interesting phenomenon is that by varying the parameters $\mu$ and $\eta$, the proposed model can not only provide the traditional decomposition result shown in Fig. 2(c), but also yield meaningful decomposition as depicted in Fig. 2(d), where the stripe of the animal are almost separated from the background and can be used for image segmentation. More supporting numerical illustrations are provided in Section 4 and the Supplemental Material. In summary, our model can be viewed as a weighted TV- $L_{1}$ model, and thus provide more freedom to deal with various image processing tasks. 


\section{Optimization Solver: ADM Method to Solve the SGTD Model}

\subsection{ADM Method to Solve the SGTD Model}

Although the SGTD regularization has exhibited some appealing properties in model setting, the issues of computational complexity and local optimality have to be addressed due to the non-convexity and high non-linearity nature of the problem, which limits its practical application. Therefore, developing an efficient and robust solver is still highly desirable. In this subsection, an augmented Lagrangian (AL) method is proposed to solve the problem.

AL method is a well studied optimization algorithm for solving the constrained problems in mathematical programming community [13]. Recently, it is enjoying a repopularization mainly due to the work of Osher et al. [21] and has been used in various applications of signal/image processing $[1,17,7,23]$. The AL related methods usually employ the operator splitting first to transform the original unconstrained minimization problem to an equivalent constrained problem, and then the alternating-minimization strategy is used to iteratively find solutions of the subproblems. Generally speaking, the AL scheme aims to solve the following problem:

$$
\begin{aligned}
& \min _{x, y, z}\left\{\frac{\mu}{2}\|z\|_{2}^{2}+\sum_{i=1}^{n^{2}}\left(\eta+\left|z_{i}\right|\right) \cdot\left\|y_{i}\right\|_{2}\right\} \\
& \text { s.t. } \quad y_{i}=D_{i} x, \quad i=1, \ldots, n^{2} \\
& z=f-x
\end{aligned}
$$

where $y_{i} \in R^{2}, i=1, \cdots, n^{2}$ and $z$ are auxiliary variables. Problem (4) can be solved via the standard augmented Lagrangian method. Specifically, letting $y=\left[y_{1}, \cdots, y_{n^{2}}\right]$, $\lambda_{1}=\left[\lambda_{11}, \cdots, \lambda_{1 n^{2}}\right]$ and starting from $\lambda^{0}=0$, it solves

$$
\begin{aligned}
& \left(x^{k+1}, z^{k+1}, y^{k+1}\right)=\arg \min _{x, z, y} \mathcal{L}\left(x, z, y, \lambda^{k}\right) \\
& =\arg \min _{x, z, y} \sum_{i=1}^{n^{2}}\left(\eta+\left|z_{i}\right|\right) \cdot\left\|y_{i}\right\|_{2}-\lambda_{1 i}^{T}\left(y_{i}-D_{i} x\right)+\frac{\mu}{2}\|z\|_{2}^{2} \\
& +\frac{\beta_{1}}{2} \sum_{i=1}^{n^{2}}\left\|y_{i}-D_{i} x\right\|^{2}-\lambda_{2}^{T}(z-x+f)+\frac{\beta_{2}}{2}\|z-x+f\|^{2}
\end{aligned}
$$

at the $k$-th iteration for $\left(x^{k+1}, y^{k+1}, z^{k+1}\right)$, then updates the mulitpliers $\lambda_{1}$ and $\lambda_{2}$ by the formula

$$
\begin{aligned}
& \lambda_{1}^{k+1}=\lambda_{1}^{k}-\beta_{1}\left(y^{k+1}-D x^{k+1}\right) \\
& \lambda_{2}^{k+1}=\lambda_{2}^{k}-\beta_{2}\left[z^{k+1}-\left(x^{k+1}-f\right)\right]
\end{aligned}
$$

Since solving the augumented Lagrangian function (5) for $x, y$ and $z$ simultaneously can be difficult, an alternative choice is to minimize it with respect to each block variable $x, y$ and $z$ one at a time while fixing the other two blocks at their latest values, and then update the Lagrange multiplier using

$$
\begin{aligned}
y^{k+1}= & \arg \min _{y}\left\{\sum_{i=1}^{n^{2}}\left(\eta+\left|z_{i}^{k}\right|\right) \cdot\left\|y_{i}\right\|_{2}\right. \\
& \left.+\frac{\beta_{1}}{2} \sum_{i=1}^{n^{2}}\left\|y_{i}-D_{i} x^{k}-\lambda_{1}^{k} / \beta_{1}\right\|^{2}\right\} \\
z^{k+1}= & \arg \min _{z}\left\{\sum_{i=1}^{n^{2}}\left(\eta+\left|z_{i}\right|\right) \cdot\left\|y_{i}^{k+1}\right\|_{2}+\frac{\mu}{2}\|z\|^{2}\right. \\
& \left.+\frac{\beta_{2}}{2}\left\|z-\left(x^{k}-f\right)-\lambda_{2}^{k} / \beta_{2}\right\|^{2}\right\} \\
x^{k+1}= & \arg \min _{x}\left\{\frac{\beta_{1}}{2} \sum_{i=1}^{n^{2}}\left\|y_{i}^{k+1}-D_{i} x-\lambda_{1}^{k} / \beta_{1}\right\|^{2}\right. \\
& \left.+\frac{\beta_{2}}{2}\left\|z-(x-f)-\lambda_{2}^{k} / \beta_{2}\right\|^{2}\right\}
\end{aligned}
$$

\section{- $y$-and $z$-subproblems}

Firstly, the minimization of Eq.(7) with respect to $y$ can be computed analytically. Concretely, we obtain the optimal solution:

$$
\begin{aligned}
y_{i}^{k+1}=\max & \left\{\left\|D_{i} x^{k}+\lambda_{1}^{k} / \beta_{1}\right\|_{2}-\left(\eta+\left|z_{i}^{k}\right|\right) / \beta_{1}, 0\right\} \\
& \cdot \frac{D_{i} x^{k}+\lambda_{1}^{k} / \beta_{1}}{\left\|D_{i} x^{k}+\lambda_{1}^{k} / \beta_{1}\right\|_{2}}
\end{aligned}
$$

Simlarily, it follows that

$$
\begin{gathered}
z^{k+1}=\operatorname{sgn}\left\{\frac{\beta_{2}}{\mu+\beta_{2}}\left(x^{k}-f+\lambda_{2}^{k}\right) / \beta_{2}\right\} \odot \max \left\{\frac{\beta_{2}}{\mu+\beta_{2}}\right. \\
\left.\cdot\left(x^{k}-f+\lambda_{2}^{k}\right) / \beta_{2}-\beta_{2}\left\|D x^{k}\right\| /\left(\mu+\beta_{2}\right), 0\right\}
\end{gathered}
$$

where sgn and $\odot$ respresent the signum and point-wise product function respectively, and both operations are implemented by compoment-wise manner.

\section{- $x$-subproblem}

By taking the derivative of Eq.(9) with respect to $x$ and setting it to zero,

$$
\begin{aligned}
& \left(\beta_{1} D^{T} D+\beta_{2}\right) x \\
= & \beta_{1} D^{T}\left(y^{k+1}-\lambda_{1}^{k} / \beta_{1}\right)+\beta_{2}\left(z+f-\lambda_{2}^{k} / \beta_{2}\right)
\end{aligned}
$$

we can get the solution $x^{k+1}$ :

$\mathcal{F}^{-1}\left\{\frac{\mathcal{F}\left(\beta_{1} D^{T}\left(y^{k+1}-\lambda_{1}^{k} / \beta_{1}\right)+\beta_{2}\left(z+f-\lambda_{2}^{k} / \beta_{2}\right)\right)}{\beta_{1} \mathcal{F}^{\star}(D) \odot \mathcal{F}(D)+\beta_{2}}\right\}$

where $\mathcal{F}$ represents the two dimensional discrete Fourier transform.

The whole SGTD mehod is summarized as follows: 


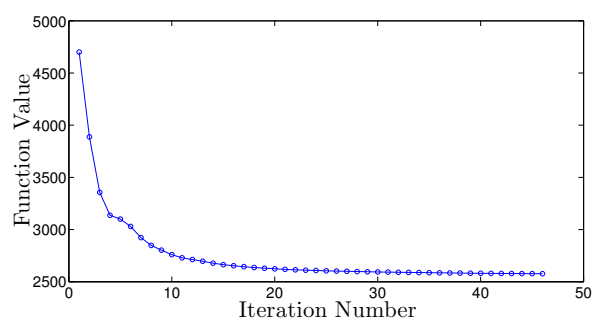

(a)
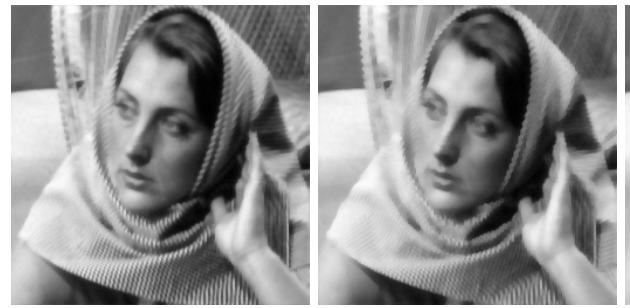

(b)

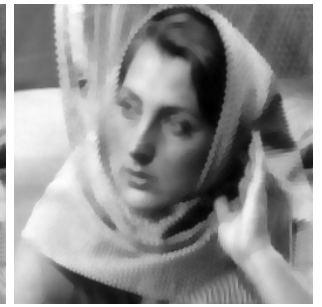

Figure 3. The plot of function values vs iteration number, and the intermediate structure images at different iterations. (a) Objective value evolution. (b) The intimidate structure image obtained by the algorithm at the 3 -th, 5 -th, and 7 -th iteration.

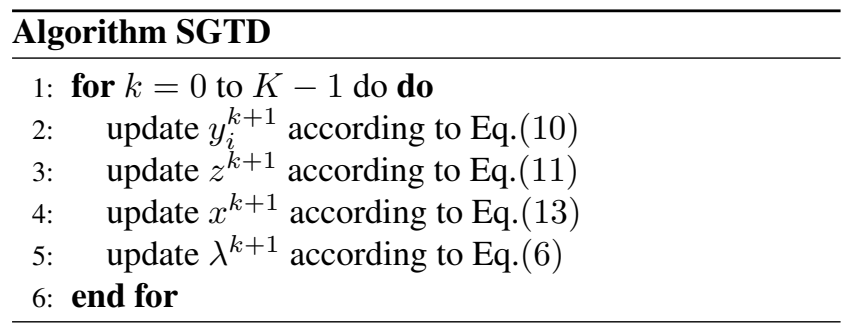

\subsection{Computation Cost and Parameter Setting}

At each iteration, the computational cost of (10) and (11) is linear with respect to the problem size, namely $O\left(n^{2}\right)$. Additionally, the main cost for solving (13) is two FFTs (inlcuding one inverse FFT), each at a cost of $O\left(n^{2} \log (n)\right)$. The proposed method contains two parameters $\beta_{1}$ and $\beta_{2}$, whose setting can be referred to [24, 19]. In all the experiments of this article, we empirically choose $\beta_{1}=5$ and $\beta_{2}=20$.

As mentioned in the literature [23, 24, 19], one advantage of the ADM based optimization is that it can decrease the objective function rapidly. This advantage can be observed from Fig. 3(a) that the objective function value changes slightly only after 20 iterations. Fig. 3(b) displays the intermediate structure images obtained at the 3-th, 5 -th, and 7 -th iteration, where we can find the proposed method quickly updates the cartoon image in iterations. It indicates the effectiveness of the alternating strategy adopted by our method.

\section{Experimental Results}

In this section, we evaluate the performance of SGTD on several experiments under the scenario of decompostion, which aims to investigate the effect of imposing structural incoherence (when working on the color images, the extension is the same as that in [19]). In the experiments, the proposed method was compared with TV- $L_{1}$ method ${ }^{1}$, RTV method ${ }^{2}$, OSV and DOSV methods [21], and the ADE

\footnotetext{
1 http: //www. caam.rice. edu/ wy1/ParaMaxFlow.

2 http://www.cse.cuhk.edu.hk/ leojia/projects/ texturesep.
}

based method $[18]^{3}$. All the test images are normalized to have a maximum magnitude of 1 . The parameter setting of all these methods follows the rule that choosing the one gives the best visual result.

\subsection{Parameter Adjustment}

In the proposed SGTD regularized model, two weights $\mu$ and $\eta$ are involved to balance the contributions of the three terms in the objective. The effect of varying parameter values is demonstrated in Fig. 4. The parameter $\mu$ takes 400, 800 and 1500 from top to bottom, and $\eta$ takes $0.001,0.003$ and 0.006 from left to right. We can see that, the textural part of the results with smaller parameters mainly contain the strips of the animal, and may favor the followed classification or segmentation tasks [3, 4]. As both parameters increasing, the results approximate to the traditional decomposition task. The well-performed separation to various types of meaningful cartoon and texture patterns under different parameter values indicates the diverse usage of the proposed method.

\subsection{Decomposition Comparison with State-of-the- art Methods on Standard Images}

When visually evaluating the performance of decomposition method, one important criterion is to consider how strong the remaining parts of one component on the image of another component [18]. For a part of the image "Barbara", we can see on Fig. 5(a) that TV- $L_{1}$ method cannot completely eliminate the texture from the table cover, while there are apparent cartoon edges on the texture image. Many other methods like OSV and DOSV provide similar results [18], which are not shown here due to the limit of space. AD-aBLMV-ADE and RTV methods can eliminate the texture from the cartoon image, but the slow changes of gray level values shown in the region of table leg are also apparent on the texture image. The proposed method successfully eliminates the texture from the cartoon while visually almost no cartoon parts appearing on the texture image

\footnotetext{
${ }^{3}$ The results using DOSV and ADE based method are copied from [18] directly for a fair comparison since the implementations are not public.
} 

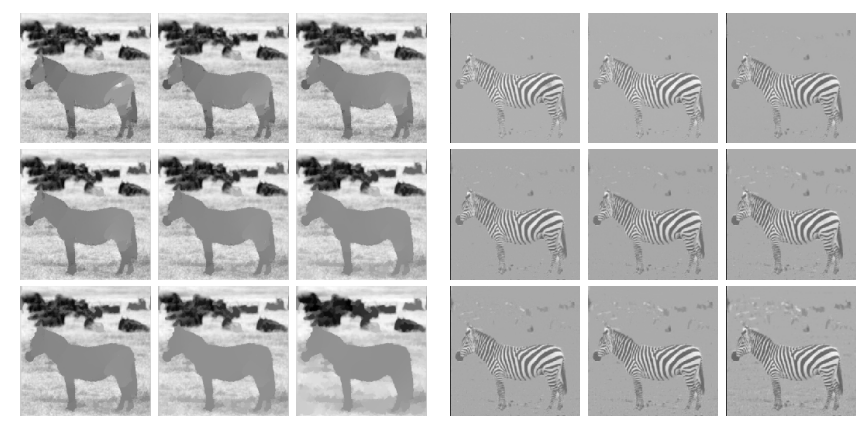

Figure 4. The effect of varying parameters. The parameter stakes 400, 800 and 1500 from top to bottom, and takes $0.001,0.003$ and 0.006 from left to right. The wellperformed separation to various types of meaningful cartoon and texture patterns indicates the diverse usage of the proposed method.

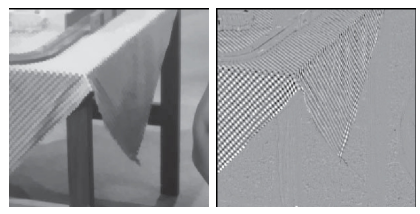

(a) $\mathrm{TV}-L_{1}$

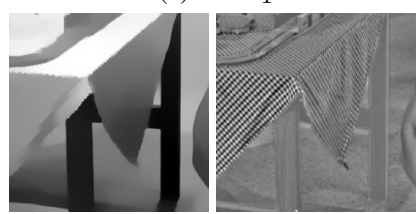

(c) RTV

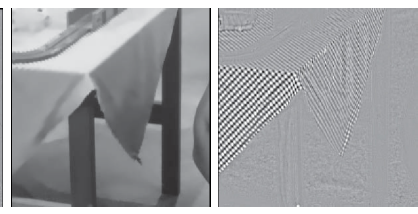

(b) AD-aBLMV-ADE[18]

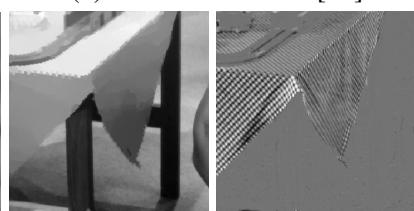

(d) SGTD
Figure 5. Comparison with state-of-the-art methods on image "Barbara".

(see Fig. 5(d)), and thus gives the best separation results and exhibits contrast-preserving feature.

Fig. 6 shows the results of decomposing the challenging image "Zebra" using different methods. It can be seen that DOSV performs worst since it cannot eliminate the larger texture parts without blurring the cartoon. RTV performs poorly with some texture parts remaining on the cartoon, while some nontextural parts such as slow changes of gray level values and the background are apparent on the texture image. It is possibly due to the limitation that RTV cannot distinguish between structure and texture that are similar in scales. AD-aBLMV-ADE performs slightly better than the RTV method by eliminating most of the texture on the animal but the nontextural parts such as the background are still apparent on the texture image. Our proposed method gives the best results visually. When applying three decomposition models OSV, TV- $L_{1}$ and SGTD to the image of "4-textures" depicted in Fig. 7, the parameter values were adjusted aiming to extract the woven texture (the upper right part) accurately. Fig. 7 exhibits the difference of the three methods in decomposition capability. In this example, TV-

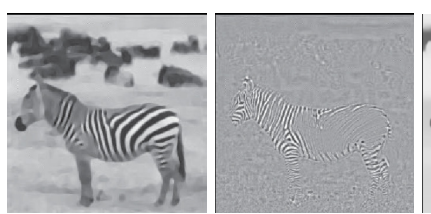

(a) DOSV[16]

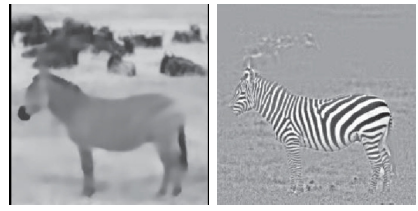

(c) AD-aBLMV-ADE[18]

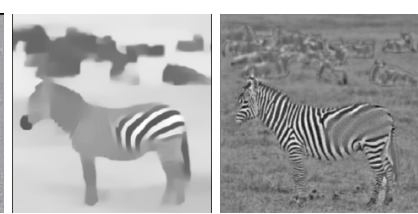

(b) RTV

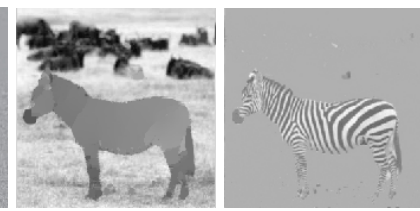

(d) SGTD
Figure 6. Comparison with state-of-the-art methods on image "Zebra".

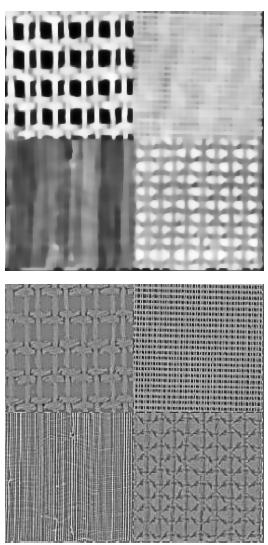

(a) OSV

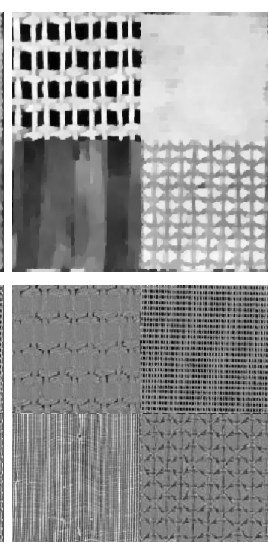

(b) TV- $L_{1}$

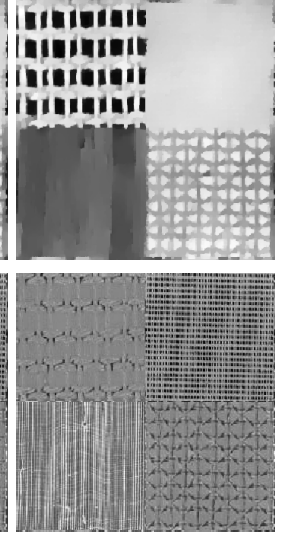

(c) SGTD
Figure 7. Comparison with state-of-the-art methods on image "4-textures".

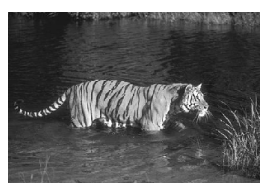

(a) Input image
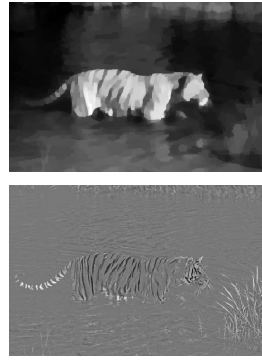

(c) TV- $L_{1}$

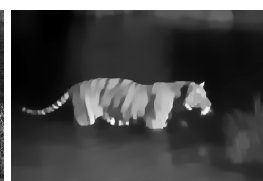

(b) RTV

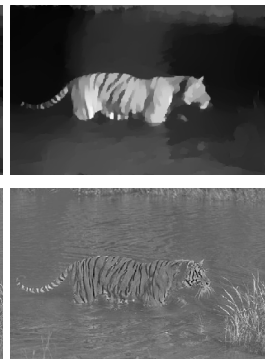

(d) SGTD
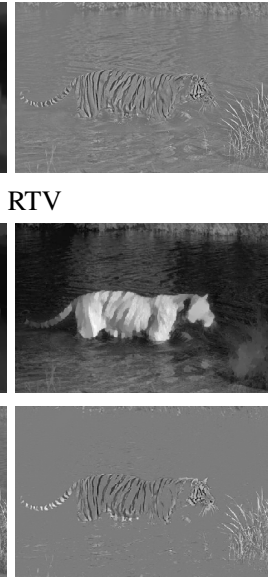

(e) SGTD
Figure 8. Comparison with state-of-the-art methods on "Tiger" image containing complex texture. The parameter pairs $(\mu, \eta)$ in (d) and (e) are $(1500,0.006)$ and $(400,0.003)$ respectively. 


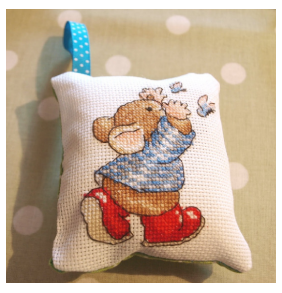

(a) Input image

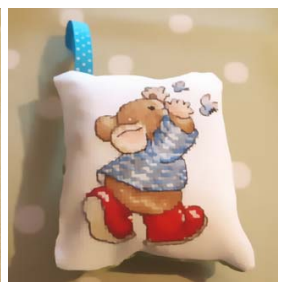

(b) RTV

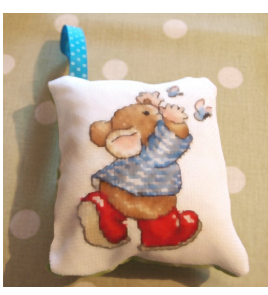

(c) SGTD
Figure 9. Comparison with RTV on image "crossstitch27".

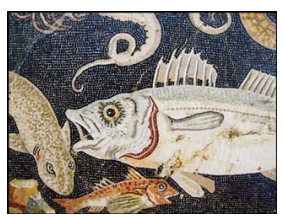

(a) Input image

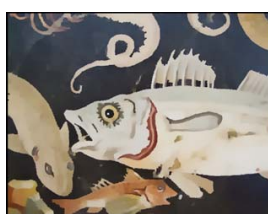

(b) RTV

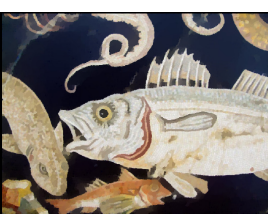

(c) SGTD
Figure 10. Comparison with RTV on "Pompeii FishMosaic".

$L_{1}$ and our SGTD overall perform at a similar level. However, in the upper right part of the test image, our method outperforms TV $-L_{1}$ by yielding a flatter and more uniform cartoon.

Fig. 8 presents the results of one image containing complex textures obtained by RTV, TV- $L_{1}$ and SGTD. Almost none of them can completely eliminate the stripes on the tiger. Compared to the results of RTV in Fig. 8(b) and TV$L_{1}$ in Fig. 8(c), the grass part in the image is better removed from the cartoon part by the proposed SGTD as shown in Fig. 8(d). Additionally, as can be seen in Fig. 8(e), the ripple can be well-separated from the texture part by tuning the parameter of our method, thus the tiger may be easier to be segmented from the image [4].

\subsection{Removing Non-uniform and Anisotropic Tex- ture}

In this subsection, three examples with non-uniform and anisotropic texture are shown to exhibit the flexibility of our method in dealing with other image processing task, which is slightly different from image decomposition but focuses on extracting main structure. Fig.9 displays the results by RTV and SGTD. It can be observed that the performance of removing texture from the cross stitch by both methods is very similar, while our method preserves more fine details than RTV on the background.

Fig. 10(a) shows a "Pompeii FishMosaic" image, in which the main structures are surrounded by the background formed by many tiles with salient but fine tessera boundaries, making the extraction very challenging [6]. Results from this method is presented in Fig. 10 (b). Although this method makes use of local signed gradients and the relative total variation (RTV) exhibits special properties, it fails to preserve some fine structures compared to our method shown in Fig.10(c). The result from our method indicates that SGTD has better capability on contrast-preserving.

Finally, we show an example where the texture is highly non-uniform and anisotropic. Fig. 11 displays the comparison between RTV and our SGTD on one image from the BSDS database [8]. It is clear that the structure parts such as of the cheek and hair of the girl are better preserved in the result of SGTD than that of RTV. Besides, the decomposition of SGTD preserves the contrast between the girl and the background better. More examples are included in the Supplemental Material.

\section{Conclusion}

In this paper, a decorrelating regularizer for extracting meaningful structure from texture was proposed. The image decomposition results were improved by forcing incoherence between the gradient magnitude of the cartoon and texture components. The proposed model can be viewed as a weighted TV- $L_{1}$ method, and thus posses the advantages of TV- $L_{1}$, such as contrast-preserving and data-driven scale selection. Additionally, the proposed model allows a high level of freedom and robustness due to the nonlinear formulation. With the aid of augmented Lagrangian and alternating direction methods, the original non-linear problem was transformed into a set of subproblems that are much easier to be solved with both accuracy and efficiency. Experimental results demonstrate the effectiveness and robustness of the proposed method compared to several state-of-the-art methods.

\section{Acknowlegements}

This work was partially supported by grant NSF (No.61102043, 61250005, 61262084, 61362001), Youth Scientic Research Foundation of Jiangxi Province 20132BAB211030 and the Basic Research Program of Shenzhen JC201104220219A.

\section{References}

[1] M. Afonso, J. Bioucas-Dias, and M. Figueiredo. An augmented lagrangian approach to the constrained optimization formulation of imaging inverse problems. Image Processing, IEEE Transactions on, 20(3):681-695, 2011. 4

[2] M. Bertalmio, L. Vese, G. Sapiro, and S. Osher. Simultaneous structure and texture image inpainting. Image Processing, IEEE Transactions on, 12(8):882-889, 2003. 1

[3] T. Chen, Y. Wotao, X. Zhou, D. Comaniciu, and T. Huang. Total variation models for variable lighting face recognition. Pattern Analysis and Machine Intelligence, IEEE Transactions on, 28(9):1519-1524, 2006. 1, 5

[4] M. Donoser, M. Urschler, M. Hirzer, and H. Bischof. Saliency driven total variation segmentation. In Computer 


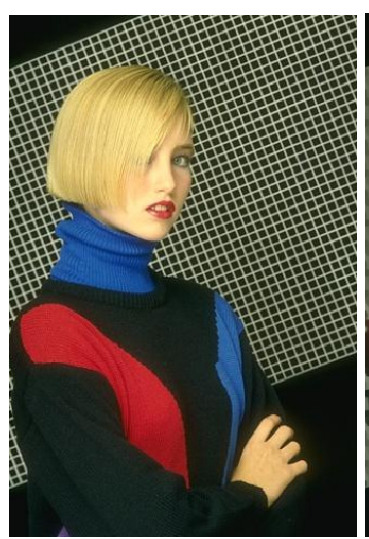

(a) Input image

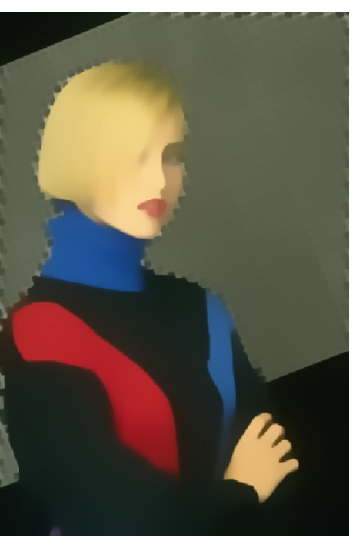

(b) RTV
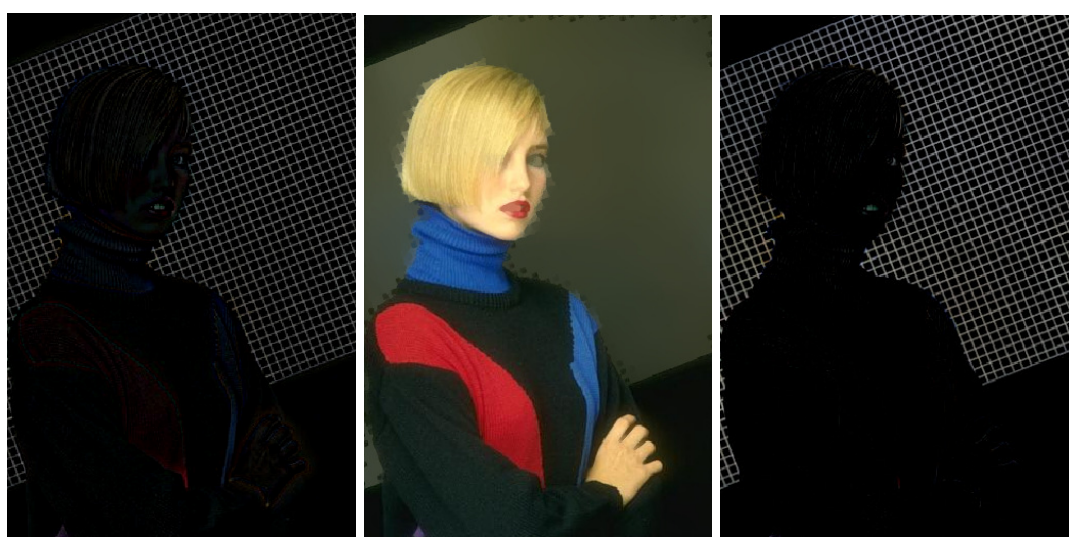

(c) SGTD

Figure 11. Comparison with state-of-the-art methods on image from BSDS database.

Vision, 2009 IEEE 12th International Conference on, pages 817-824, 2009. 5, 7

[5] Z. Farbman, R. Fattal, D. Lischinski, and R. Szeliski. Edgepreserving decompositions for multi-scale tone and detail manipulation. ACM Trans. Graph., 27(3):67, 2008. 1

[6] X. Li, Y. Qiong, X. Yang, and J. Jiaya. Structure extraction from texture via relative total variation. ACM Transactions on Graphics (TOG), 31(6):139, 2012. 2, 7

[7] Q. Liu, S. Wang, and J. Luo. An multi-scale augmented lagrangian approach to general dictionary learning for image denosing. Journal of Visual Communication and Image Representation, 23:753-766, 2012. 4

[8] D. Martin, C. Fowlkes, D. Tal, and J. Malik. A database of human segmented natural images and its application to evaluating segmentation algorithms and measuring ecological statistics. In Computer Vision, 2001 IEEE Eighth International Conference on, volume 2, pages 416-423, 2001. 7

[9] Y. Meyer. Oscillating patterns in image processing and nonlinear evolution equations: the fifteenth Dean Jacqueline B. Lewis memorial lectures, volume 22. Amer Mathematical Society, 2001. 1

[10] M. Nikolova. A variational approach to remove outliers and impulse noise. Journal of Mathematical Imaging and Vision, 20(1-2):99-120, 2004. 1

[11] S. Osher, A. Solé, and L. Vese. Image decomposition and restoration using total variation minimization and the $l_{1}$ norm. Multiscale Modeling \& Simulation, 1(3):349-370, 2003. 1

[12] D. C. Paquin, D. Levy, E. Schreibmann, and L. Xing. Multiscale image registration. Mathematics, page 5, 2006. 1

[13] R. T. Rockafellar. Augmented lagrangians and applications of the proximal point algorithm in convex programming. Mathematics of operations research, 1(2):97-116, 1976. 4

[14] L. I. Rudin, S. Osher, and E. Fatemi. Nonlinear total variation based noise removal algorithms. Physica D: Nonlinear Phenomena, 60(1):259-268, 1992. 1

[15] T. Saito, Y. Ishii, H. Aizawa, D. Yamada, and T. Komatsu. Image-processing approach via nonlinear imagedecomposition for a digital color camera. In Image Pro- cessing, IEEE International Conference on, pages 905-908, 2008. 1

[16] R. Shahidi and C. Moloney. Decorrelating the structure and texture components of a variational decomposition model. Image Processing, IEEE Transactions on, 18(2):299-309, 2009. 2, 6

[17] Y. Shen, Z. Wen, and Y. Zhang. Augmented lagrangian alternating direction method for matrix separation based on low-rank factorization. Optimization Methods and Software, pages $1-25,2012.4$

[18] D. Szolgay and T. Szirányi. Adaptive image decomposition into cartoon and texture parts optimized by the orthogonality criterion. Image Processing, IEEE Transactions on, 21(8):3405-3415, 2012. 2, 5, 6

[19] M. Tao and J. Yang. Alternating direction algorithm for total variation deconvolution in image deconstrution. available at Optimization Online. 5

[20] L. A. Vese and S. J. Osher. Modeling textures with total variation minimization and oscillating patterns in image processing. Journal of Scientific Computing, 19(1-3):553-572, 2003. 1

[21] Y. Wotao, S. Osher, D. Goldfarb, and J. Darbon. Bregman iterative algorithms for $\ell_{1}$-minimization with applications to compressed sensing. SIAM Journal on Imaging Sciences, 1(1):143-168, 2008. 4, 5

[22] L. Xu, C. Lu, Y. Xu, and J. Jia. Image smoothing via $\ell_{0}$ gradient minimization. ACM Transactions on Graphics (SIGGRAPH Asia), 2011. 1

[23] J. Yang and Y. Zhang. Alternating direction algorithms for $\ell_{1}$-problems in compressive sensing. SIAM journal on scientific computing, 33(1):250-278, 2011. 4, 5

[24] J. Yang, Y. Zhang, and W. Yin. An efficient TVL1 algorithm for deblurring multichannel images corrupted by impulsive noise. SIAM Journal on Scientific Computing, 31(4):28422865, 2009. 5

[25] W. Yin, D. Goldfarb, and S. Osher. Image cartoon-texture decomposition and feature selection using the total variation regularized 11 functional. In Variational, Geometric, and Level Set Methods in Computer Vision, pages 73-84. Springer, 2005. 1, 3 